\title{
PLANE-STRAIN CRACK-TIP STRESS FIELD FOR ANISOTROPIC PERFECTLY-PLASTIC MATERIALS
}

\author{
J. PAN \\ Department of Mechanical Engineering and Applied Mechanics, The University of Michigan, \\ Ann Arbor, MI 48109, U.S.A.
}

(Received 29 January 1986)

\begin{abstract}
Plane-STRAIN crack-tip stress solutions for anisotropic perfectly-plastic materials are presented. These solutions are obtained using the plane-strain slip-line theory developed by Rice (1973). The plastic anisosotropy is described by the Hill quadratic yield condition. The crack-tip stress solutions under symmetric (Mode I) and anti-symmetric (Mode II) conditions agree well with the low-hardening solutions for the corresponding power-law hardening materials. The crack-tip stress solutions under mixed Mode I and II conditions are also presented. All the solutions indicate that the general features of the slip-line field near a crack tip in orthotropic plastic materials with the elliptical yield contours in the Mohr plane are the same as those associated with isotropic plastic materials. However, the angular variations of the crack-tip stress fields for the materials with large plastic orthotropy differ substantially from those for isotropic plastic materials. Modifications due to polygonal yield contours are outlined and implications of solutions to the fracture analysis of ductile composite materials containing macroscopic flaws are discussed.
\end{abstract}

\section{INTRODUCTION}

Plastic anisotropic behavior of engineering materials may be due to their crystallographic microstructure and/or prior plastic deformation as the result of manufacturing processes. However anisotropic behavior of engineering composite materials is mainly due to fiber reinforcement in the materials.

Cracks or flaws, still, may exist in these anisotropic engineering materials. In order to assure the structural integrity of the materials, an investigation of the stress and strain fields near a crack tip in the materials is necessary.

HUTCHINSON $(1968 a, b)$ and RICE and ROSENGREN (1968) presented the strainhardening and perfectly-plastic solutions of the crack-tip field for isotropic materials under pure Mode I and pure Mode II conditions. SHIH $(1973,1974)$ presented the strain-hardening and perfectly-plastic solutions of the crack-tip field for isotropic materials under mixed Mode I and II conditions. HAYASHI (1979) and PAN and SHIH (1986) presented the strain-hardening solutions of the singular crack-tip field for orthotropic materials under pure Mode I and pure Mode II conditions.

In this paper, the plane-strain crack-tip stress solutions for anisotropic perfectlyplastic materials are presented. The solutions are constructed using the plane-strain slip-line theory of RICE (1973) for arbitrary anisotropic rigid-plastic materials. The 
quadratic yield function introduced by HiLl $(1948,1950)$ is used to describe anisotropic plastic behavior. Note that the yield surface based on the quadratic function is smooth. However, for materials with highly oriented microstructure such as single crystals, the yield surfaces have corners and flats when discrete crystalline slip is considered to be the primary mechanism for plastic deformation (BISHOP and HILL, 1951). RICE (1973) suggested that we can use a simple polygon as the yield contour to describe approximately the plastic behavior of ductile composite materials under plane-strain conditions. Therefore we also discuss the crack-tip stress solutions for the materials with polygonal yield contours and the implications of these crack-tip stress solutions to the fracture analysis of ductile composite materials.

\section{The Rice Plane-strain Slip-Line Theory}

The plane-strain slip-line theory for isotropic rigid-plastic materials is well known, for example, see Hill (1950). HiLl (1950) extended the theory to include plastic anisotropy for incompressible materials with ellipsoidal yield surfaces. RICE (1973) generalized the theory for incompressible materials with arbitrary anisotropic convex yield contours. By employing the Rice method which is remarkably simple, we can construct the stress field near the tip of a crack in anisotropic perfectly-plastic materials.

Here we briefly discuss the plane-strain slip-line theory of RICE (1973). Rice considered a plane-strain deformation in the $x, y$ plane as shown in Fig. $1 \mathrm{~b}$. Denote $\sigma_{x}, \sigma_{y}$ and $\sigma_{x y}$ as the associated in-plane normal stresses and shear stress. Rice showed that under plane-strain conditions incompressible rigid-plastic materials, with arbitrary anisotropy, in which the plastic deformation complies with the principle of maximum plastic work, have a reduced yield criterion which is a function of $\left(\sigma_{x}-\sigma_{y}\right) / 2$ and $\sigma_{x y}$. In Fig. 1a, an arbitrary but smooth yield contour is plotted in the Mohr plane in terms of $\left(\sigma_{x}-\sigma_{y}\right) / 2$ and $\sigma_{x y}$. Denote the orientation of the outward normal at a point $P^{*}$ on the yield contour by $2 \phi$ which is measured counterclockwise from the direction of the $\sigma_{x y}$ axis. Then by considering the maximum plastic work inequality and equilibrium conditions, two orthogonal families of slip-line, labelled $\alpha$ and $\beta$, about a point $P$ (which corresponds to point $P^{*}$ on the yicld contour) in the plastic region of the $x, y$ plane as shown in Fig. $1 \mathrm{~b}$ can be constructed. The mesh of the sliplines is defined such that the counterclockwise angle between the $\alpha$ lines through point $P$ and the $x$ direction is $\phi$. The integrated equations of stress equilibrium become

$$
\begin{array}{ll}
\sigma-l=\text { constant } & \text { along an } \alpha \text { line } \\
\sigma+l=\text { constant } & \text { along a } \beta \text { line }
\end{array}
$$

where $\sigma$ is the mean in-plane stress $\left(=\left(\sigma_{x}+\sigma_{y}\right) / 2\right)$, and $l$ represents the arc length around the yield contour to point $P$, increasing counterclockwise as shown in Fig. 1a.

When there is a corner on the yield surface, there is a jump in $\phi$ at the corner. Therefore the direction of the $\alpha$ lines associated with the corner has a jump, When there is a flat segment on the yield surface, a stress discontinuity may arise. Further details of the slip-line theory for anisotropic plastic materials can be found in RICE (1973). 


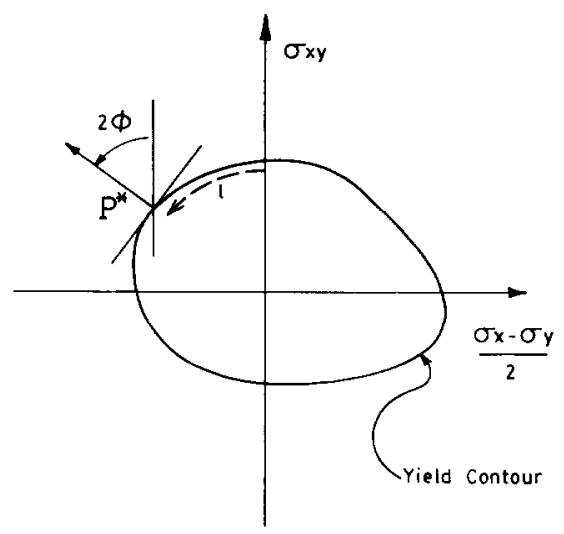

(a)

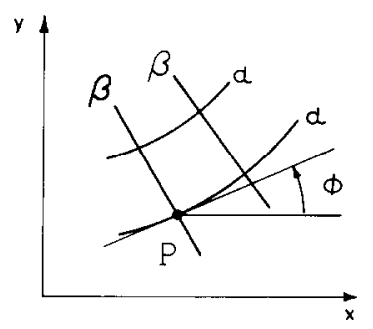

(b)

FIG. 1. (a) A yield contour in the Mohr plane. (b) The slip-line field in the physical plane.

\section{Crack-tip Stress Field for Orthotropic Plastic Materials}

Consider a plane-strain problem of a crack in an anisotropic solid as shown in Fig. 2. The crack tip is located at the origin of the Cartesian coordinate system $x, y$. The polar coordinates $r, \theta$ with respect to the crack tip are shown in Fig. 2. Note that the Cartesian coordinate $z$ is perpendicular to the $x y$ plane. For a material with the plastic behavior described by the Hill quadratic yield condition, when the symmetry axes of

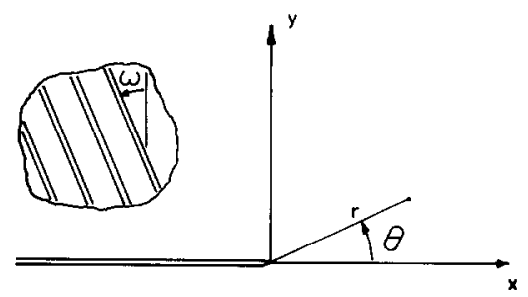

FIG. 2. A crack in an anisotropic solid with the insert indicating the direction of fiber reinforcement for the composite material. 
the orthotropy, $X_{1}, X_{2}$ and $X_{3}$, coincide with the $x, y, z$ axes, the plane-strain yield condition can be written (HUL, 1948, 1950; PAN and SHH, 1986) as

$$
\sigma_{e}^{2} / 3=\tau_{e}^{2}=p\left(\left(\sigma_{x}-\sigma_{y}\right) / 2\right)^{2}+\left(\sigma_{x y}\right)^{2}
$$

where $\sigma_{\varepsilon}$ is the generalized effective tensile stress, $\tau_{e}$ is the generalized effective shear stress, and $p$ is a material constant. The yield condition is written to be consistent with the crack-tip field study for power-law hardening materials by PAN and SFIH (1986).

As shown in Fig. 3a, the yield condition of (3.1) is plotted as an ellipse in the Mohr plane. The ellipse is symmetric with respect to the $\sigma_{x y}$ and $\left(\sigma_{x}-\sigma_{x}\right) / 2$ axes. When $p<1$, the semimajor axis and the semiminor axis of the ellipse, denoted as $a$ and $b$, respectively, are shown in Fig. 3a, where $a=\tau_{k} / p^{1 / 2}$ and $b=\tau_{x^{*}}$. When $p=1$, equation (3.1) represents a circular yield contour in the Mohr plane and represents the planestrain yield condition for isotropic plastic materials. However, as discussed in HILL (1950) and PAN and SHIH (1986), plastic materials with orthotropic structural symmetry can still have the plane-strain isotropic yield condition. When $p>1$, the semimajor axis will be in the $\sigma_{x y}$ direction and the semiminor axis in the $\left(\sigma_{x}-\sigma_{y}\right) / 2$ direction.

As discussed in RICE (1968), slip-line theory may be used to construct the cracktip stress for elastic perfectly-plastic materials under small-scale yielding conditions.
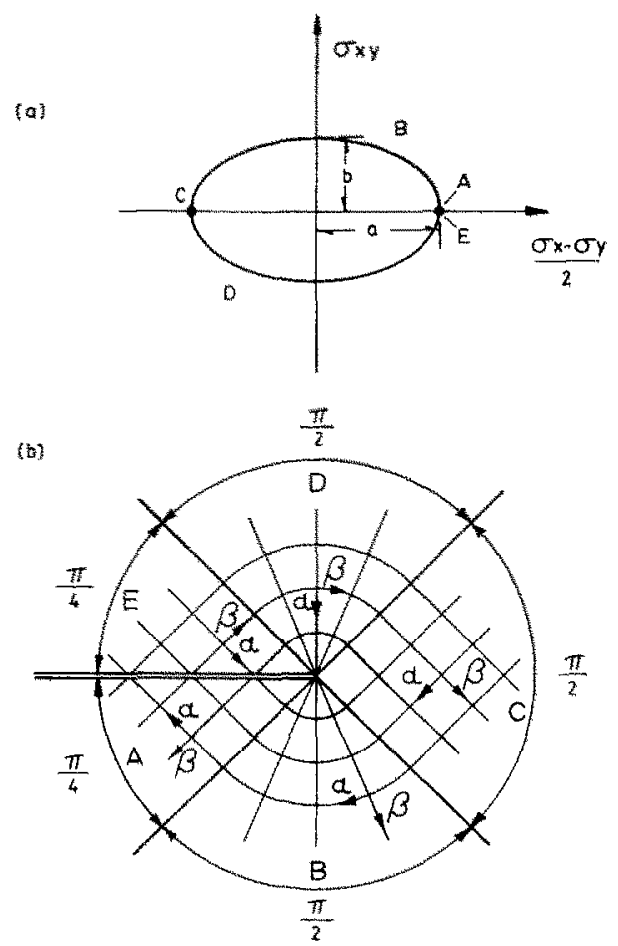

FtG. 3. (a) The elliptical yield contour. (b) The Mode I crack-tip sip-line field. 
When we consider a stationary crack in a perfectly-plastic material under small-scale yielding conditions, the stresses near the crack tip as $r \rightarrow 0$ can be treated as functions of $\theta$ only under the assumption that the material surrounding the crack tip is fully yielded. Note that due to boundedness of the $J$ integral, the displacement gradient is required (RICE, 1968) to have a $1 / r$ singularity as $r \rightarrow 0$. We here use the Rice planestrain slip-line theory, as summarized above, to construct possible crack-tip stress solutions for the perfectly-plastic material with the yield condition of equation (3.1).

\section{Mode I crack-tip field}

We first examine the Mode I crack-tip stress field for the orthotropic plastic material. The stress distribution of the Mode I plane-strain near-tip field for isotropic perfectlyplastic material under small-scale yielding conditions was presented by RICE (1968), HutCHINSON $(1968 \mathrm{a}, \mathrm{b})$ and RICE and ROSENGREN (1968). The near-tip field has the slip-line field similar to the Prandtl field which is the field under a flat-ended, rigid, frictionless punch on a half plane of isotropic rigid-plastic material. RICE (1973) showed the stress distribution under such a punch on a half plane of anisotropic rigidplastic material. When we assume that the material surrounding the crack tip is fully yielded, we can construct the Mode I crack-tip stress ficld according to the general procedure to construct the solution of the punch problem for anisotropic plastic materials as in Rice (1973). Then we compare the crack-tip stress solutions for perfectly-plastic materials with the low-hardening crack-tip stress solutions for the corresponding power-law hardening materials presented in PAN and SHIH (1986).

The stress-free boundary on the upper and lower faces of the crack requires that $\sigma_{x y}=\sigma_{y}=0$ at $\theta=-180^{\circ}$ and $\theta=180^{\circ}$. For the symmetric Mode I stress field, we consider the case that $\sigma_{x}>0$ on the stress-free crack faces. Therefore, as shown in Fig. 3a, the stress state on the stress-free crack faces must correspond to the stress state of point $A$ (or $E$ ) because $\left(\sigma_{x}-\sigma_{y}\right) / 2>0$. Note that both point $A$ and point $E$ represent the same stress state. However, the angle of the normal for point $E, 2 \phi$, is larger than that of point $A$ by $2 \pi$.

In front of the crack tip, the shear stress $\sigma_{x y}$ at $\theta=0^{\circ}$ must vanish for the symmetric Mode I stress field. Therefore, the stress state directly ahead of the crack tip could correspond to the stress state of point $C$ as shown in Fig. 3a because we anticipate that $\sigma_{y}>\sigma_{x}$ ahead of the crack tip. By examining the crack-tip stress of the Prandtl field for isotropic plastic materials and the solution of the punch problem for general anisotropic plastic materials in RICE (1973), the stress field around the crack tip can be constructed as follows.

As shown in Fig. 3b, we have the constant stress sector A with the angle of span of $\pi / 4$ below the lower crack face. The constant stress sector A corresponds to the stress state at point $A$ on the yield contour as shown in Fig. 3a and has the $\alpha$ lines with the angle of $\phi=3 \pi / 4$ as shown in Fig. 3b.

Adjacent to sector $\mathrm{A}$ we have the fan sector $\mathrm{B}$ with the angle of span of $\pi / 2$. The fan sector B represents the stress state along the yield contour from point $A$ to point $C$ counterclockwise as shown in Fig. 3a. This fan sector B has the $\alpha$ lines with the angle $\phi$ increasing from $\phi=3 \pi / 4$ to $\phi=5 \pi / 4$ as shown in Fig. $3 b$.

Adjacent to sector B and ahead of the crack tip we have the constant stress sector 
C corresponding to the stress state of point $C$ on the yield contour as shown in Fig. $3 a$. This sector $\mathrm{C}$ has the angle of span of $\pi / 2$ as shown in Fig. 3b. The $\alpha$ lines in sector $\mathrm{C}$ have the angle of $\phi=5 \pi / 4$ as shown in Fig. $3 b$.

By symmetry, we again have the fan sector $D$ with the angle of span of $\pi / 2$. The fan sector $D$ represents the stress state along the yield contour from point $C$ to point $E$ counterclockwise as shown in Fig. 3a. The $\alpha$ lines of the fan sector D have the angle $\phi$ increasing from $\phi=5 \pi / 4$ to $\phi=7 \pi / 4$ as shown in Fig. $3 b$.

Finally we have the constant stress sector $E$ with the angle of span of $\pi / 4$. The constant stress sector $\mathrm{E}$ corresponds to the stress state of point $E$ on the yield contour as shown in Fig. 3a and the $\alpha$ lines in this sector have the angle $\phi=7 \pi / 4$ as shown in Fig. $3 b$.

Employing the methodology of RICE (1973), the solutions of the crack-tip stress field have been obtained for the materials with $p=10,2,1,0.5$ and 0.1 . The yield contour for $p=1$ is circular in the Mohr plane and it represents isotropic plastic behavior. The yield contours for $p=10$ and 2 are ellipses with the semimajor axis in the $\sigma_{x y}$ direction in the Mohr plane. The yield contours for $p=0.1$ and 0.5 are ellipses with the semimajor axis in the $\left(\sigma_{x}-\sigma_{y}\right) / 2$ direction in the Mohr plane. The yield contours for $p=0.5$ and 2 represent mildly orthotropic plastic behavior and the yield contours for $p=0.1$ and 10 represent strongly orthotropic plastic bchavior.

The solutions of the crack-tip stress field for $p=10,2,1,0.5$ and 0.1 are plotted in Figs. $4 a-e$, respectively. Note that the stresses shown in Figs. $4 a-c$ are normalized by $\sigma_{f}$ and the stresses labelled with the "bar" shown in ligs. $4 \mathrm{~d}$ and $\mathrm{e}$ are normalized by $\sigma_{e} / p^{1 / 2}$. The results for $p=1$ shown in Fig $4 \mathrm{c}$ are the crack-tip stresses for isotropic plastic materials or for orthotropic plastic material with the plane-strain isotropic yield condition. The results for $p=1$ agree with the well-known Prandtl field solution presented in HUTCHINSON (1968a) and RICE and ROSENGREN (1968). It is interesting to note that the crack-tip slip-line fields for the orthotropic plastic materials with elliptical yield contours in the Mohr plane are the same as the Prandtl field associated with isotropic plastic materials. However, as shown in Figs. 4a-e, as the $p$ deviates substantially from 1 , the angular variations of the stresses deviate significantly from those for $p=1$.

Note that, because of the hyperbolic nature of the governing equations, the solutions which we obtained here are not unique. In the study of PAN and SHIH (1986), solutions of the crack-tip field for orthotropic power-law hardening materials with the yield criterion of equation (3.1) were obtained. The crack-tip stress and strain solutions for power-law hardening materials with the same five $p$ 's are shown in Figs. 2 and 3 in PAN and SHIH (1986). Comparisons of the solutions for power-law hardening materials with the solutions for perfectly-plastic materials shown in Fig. 4 in the present paper suggest that the present solutions for rigid-plastic materials indeed correspond to the perfectly-plastic limit of the solutions for the power-law hardening materials.

\section{Mode II crack-tip field}

Now we construct the anti-symmetric Mode II stress field. The stress-free condition on the crack faces requires that $\sigma_{x y}=\sigma_{y}=0$ at $\theta=-180^{\circ}$ and $\theta=180^{\circ}$. We consider 

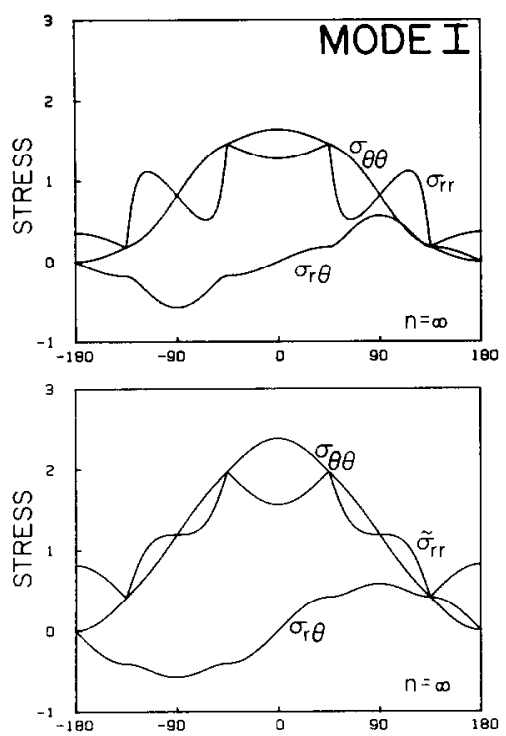

(a)

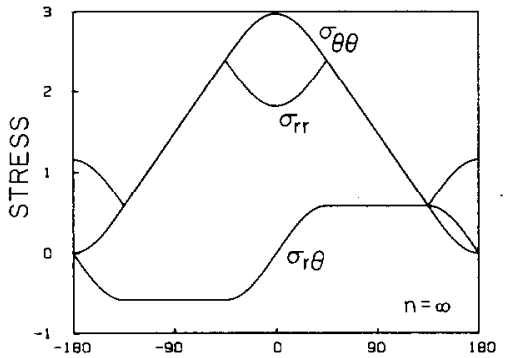

(b)

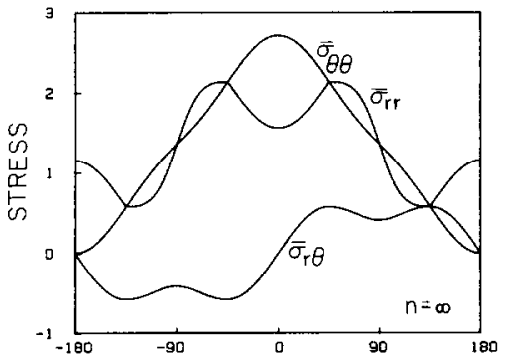

(c)

(d)

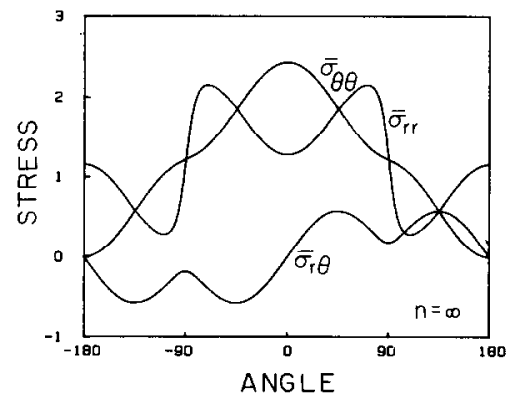

(e)

Fig. 4. The solutions of the Mode I crack-tip stress field; (a) $p=10$, (b) $p=2$, (c) $p=1$, (d) $p=0.5$, (c) $p=0.1$. 
the loading condition which produces the anti-symmetric Mode II stress field near the crack tip with $\sigma_{x}>0$ at $\theta=-180^{\circ}$ and $\sigma_{x}<0$ at $\theta=180^{\circ}$. The stress state on the lower face of the crack $\left(\theta=-180^{\circ}\right)$ therefore can correspond to the stress state of point $A$ on the yield contour as shown in Fig. 5a. The stress state on the upper face of the crack $\left(\theta=180^{\circ}\right)$ can correspond to the stress state at point $G$ on the yield contour as shown in Fig. 5a.

Directly ahead of the crack tip $\left(\theta=0^{\circ}\right)$, the normal stresses $\sigma_{x}$ and $\sigma_{y}$ should vanish for the anti-symmetric Mode II stress field. Therefore, the stress state ahead of the crack tip can possibly correspond to the stress state of point $D^{\prime}$ on the yield contour as shown in Fig. 5a. By examining the Mode II crack-tip slip-line field for isotropic plastic materials presented in HUTCHINSON $(1968 \mathrm{~b})$ and SHIH $(1973,1974)$, the stress field around the crack tip for the orthotropic plastic materials can be constructed as follows.

As shown in Fig. 5b, we have the constant stress sector A with the angle of span of $\pi / 4$ below the lower crack face. The constant stress sector A corresponds to the stress state of point $A$ on the yield contour as shown in Fig. 5a. The sector has the $\alpha$ lines with the angle of $\phi=3 \pi / 4$ as shown in Fig. $5 b$.

(c)
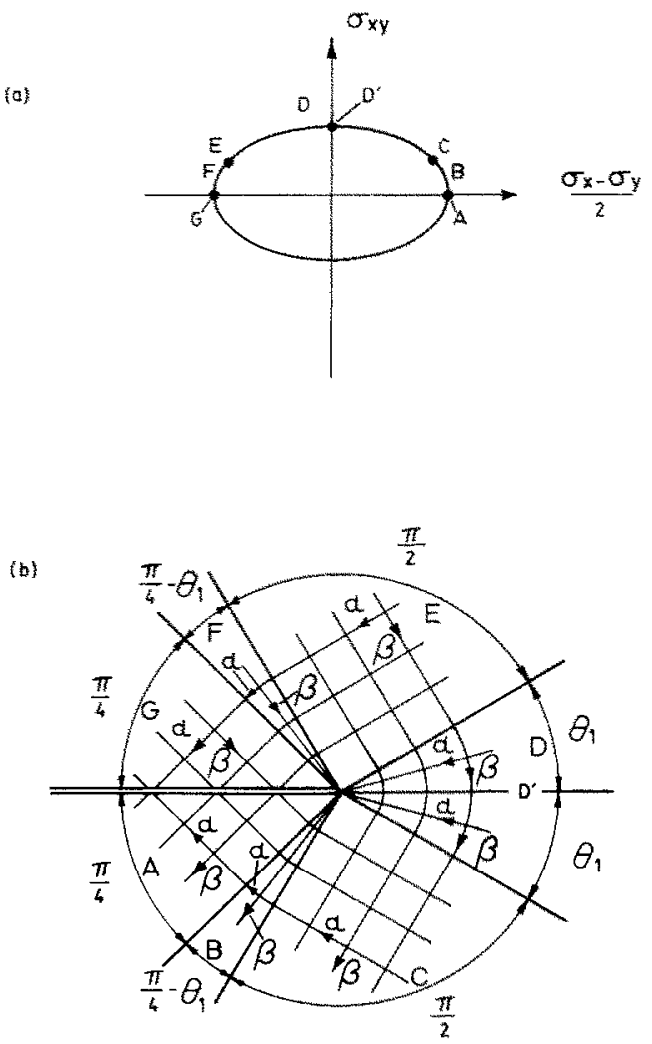

Fio. 5. (a) The elliptical yield contowr. (b) The Mode II crack-tip slip-line field. 
Adjacent to sector A we have the fan sector B with the angle of span of $\pi / 4-\theta_{1}$. The fan sector $\mathrm{B}$ represents the stress state along the yield contour from point $A$ to point $C$ counterclockwise as shown in Fig. 5a. This fan sector B has the $\alpha$ lines with the angle $\phi$ increasing from $\phi=3 \pi / 4$ to $\phi=\pi-\theta_{1}$ as shown in Fig. $5 \mathrm{~b}$. The angle $\theta_{1}$ is determined by consideration of the continuity of the stress state and the slip-line field as shown in Fig. 5 b.

Adjacent to sector B we have the constant stress sector $\mathrm{C}$ corresponding to the stress state of point $C$ on the yield contour. This sector $C$ has the angle of span of $\pi / 2$. The $\alpha$ lines in sector $C$ have the angle of $\phi=\pi-\theta_{1}$ as shown in Fig. 5b.

Adjacent to sector $\mathrm{C}$ we have the fan sector $\mathrm{D}$ with the angle of span of $2 \theta_{1}$. The fan sector $\mathrm{D}$ represents the stress state along the yield contour from point $C$ to point $E$ counterclockwise as shown in Fig. 5a. This fan sector D has the $\alpha$ lines with the angle $\phi$ increasing from $\phi=\pi-\theta_{1}$ to $\phi=\pi+\theta_{1}$ as shown in Fig. $5 \mathrm{~b}$. Because the direction of the normal at point $D^{\prime}$ has $\phi=\pi$, the stress state at $\theta=0^{\circ}$ where $\phi=\pi$ corresponds to the stress state of point $D^{\prime}$ on the yield contour.

By anti-symmetry of the Mode II stress field, we can then construct the rest of the slip-line field. Adjacent to the fan sector D we have the constant stress sector E corresponding to the stress state of point $E$ on the yield contour. Due to anti-symmetry of the Mode II stress field point $C$ and point $E$ are symmetric with respect to the $\sigma_{x y}$ axis as shown in Fig. 5a. Sector $\mathrm{E}$ has the angle of span of $\pi / 2$ and the $\alpha$ lines of the sector have the direction $\phi=\pi+\theta_{1}$.

Then we have the fan sector $F$ with the angle of span of $\pi / 4-\theta_{1}$. The fan sector F represents the stress state along the yield contour from point $E$ to point $G$ counterclockwise as shown in Fig. 5a. This fan sector F has the $\alpha$ lines with the angle $\phi$ increasing from $\phi=\pi+\theta_{1}$ to $\phi=5 \pi / 4$ as shown in Fig. 5b.

Finally we have the constant stress sector $G$ with the angle of span of $\pi / 4$. The constant stress sector $\mathrm{G}$ corresponds to the stress state of point $G$ on the yield contour as shown in Fig. 5a and the $\alpha$ lines in this sector have the angle $\phi=5 \pi / 4$ as shown in Fig. $5 b$.

The solutions of the crack-tip stress field are obtained and plotted in Figs. 6a-e for $p=10,2,1,0.5$ and 0.1 , respectively. As for the Mode I solutions, the stresses shown in Figs. 6a-c are normalized by $\sigma_{e}$ and the stresses labelled with the "bar" shown in Figs. $6 \mathrm{~d}$ and e are normalized by $\sigma_{e} / p^{1 / 2}$. The results shown in Fig. $6 \mathrm{c}$ are the crack-tip stresses for isotropic plastic materials and agree well with the results in HUTCHINSON (1968b) and SHIH $(1973,1974)$. As shown in Fig. 5b the general features of the cracktip slip-line fields for the orthotropic plastic materials with elliptical yield contours in the Mohr plane are the same as those for isotropic plastic materials with the circular yield contour in the Mohr plane. The values of $\theta_{1}$ for $p=10,2,1,0.5$ and 0.1 are $41.2^{\circ}, 38.3^{\circ}, 36.8^{\circ}, 35.2^{\circ}$ and $31.50^{\circ}$, respectively. As shown in Figs. $6 \mathrm{a}-\mathrm{e}$, when plastic orthotropy becomes large ( $p$ deviates significantly from 1 ), the angular variations of the crack-tip stresses differ substantially from those of the solutions for $p=1$.

Comparisons of the solutions of the crack-tip stress for $p=10,2,1,0.5$ and 0.1 as shown in Figs. 6a-e with the solutions of the crack-tip stress field for power-law hardening materials as reported in Figs. 6 and 7 in PAN and SHIH (1986) suggest that the present solutions for rigid-plastic materials indeed correspond to the perfectlyplastic limit of the solutions for the power-law hardening materials. 


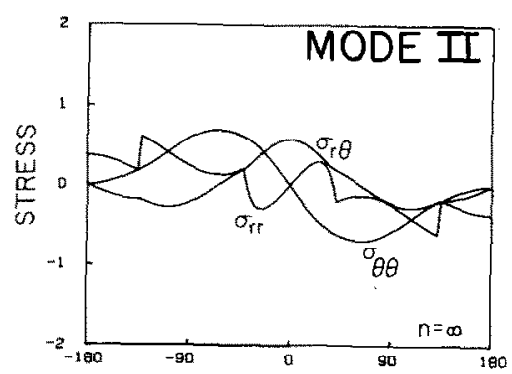

(a)

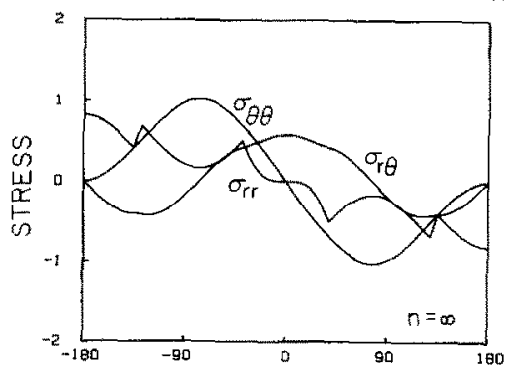

(b)

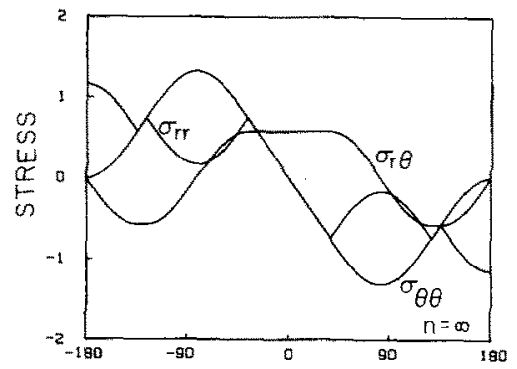

(c)

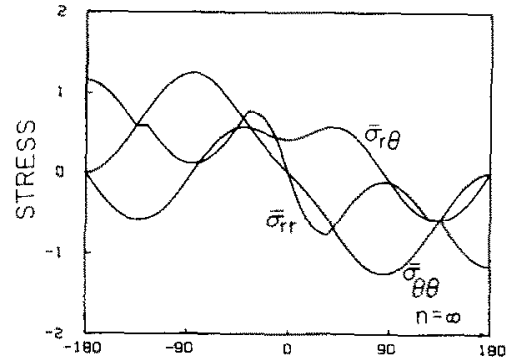

(d)

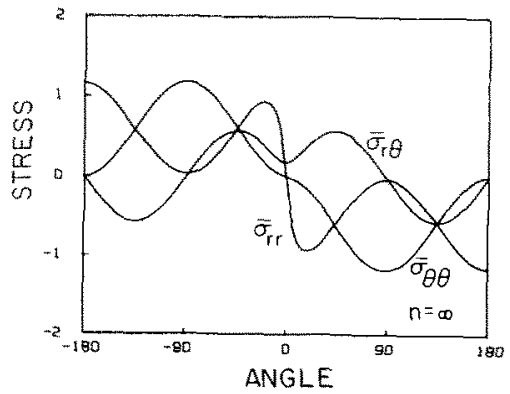

(e)

FIG. 6. The solutions of the Mode II crack-tip stress field; (a) $p=10$, (b) $p=2$, (c) $p=1$, (d) $p=0.5$, (e) $p=0.1$. 


\section{Mixed mode crack-tip field}

The study of BumIANSKY and RICE (1973) indicates that due to the boundedness of the conservation integrals the ratio of the dominant singularity of $\sigma_{x}$ below and above the crack line must be either +1 or -1 for all mixed Mode I and II cases. The ratio is +1 for Mode I and -1 for Mode II. The study of SHIH $(1973,1974)$ strongly suggests that for any amount of deviation from Mode I to mixed mode the ratio will jump from +1 to -1 for isotropic power-law hardening materials. Also, as indicated in the isotropic perfectly-plastic solutions in SHIH $(1973,1974)$, the ratio of $\sigma_{x}$ below and above the crack line jumps from +1 to -1 for any deviation from Mode I to mixed mode. By examining the mixed mode crack-tip stress field of SHIH $(1973,1974)$ for isotropic plastic materials and the present solutions of the crack-tip field under pure Mode I and pure Mode II conditions, we can construct the mixed Mode I and II stress field for the orthotropic plastic materials.

On the lower crack face, the stress-free condition requires that $\sigma_{x y}=\sigma_{y}=0$ at $\theta=-180^{\circ}$. We consider the loading condition which produces $\sigma_{x}>0$ at $\theta=-180^{\circ}$. The stress state at $\theta=-180^{\circ}$ thus corresponds to the stress state of point $A$ as shown in Fig. 7a. As suggested from the Shih solutions $(1973,1974)$ for isotropic plastic materials we assume that for any deviation from Mode I stress field we will have $\sigma_{x}<0$ at $\theta=180^{\circ}$ on the upper crack face. The stress state at $\theta=180^{\circ}$ on the upper crack face therefore corresponds to the stress state of point $G$ as shown in Fig. $7 \mathrm{a}$.

(a)
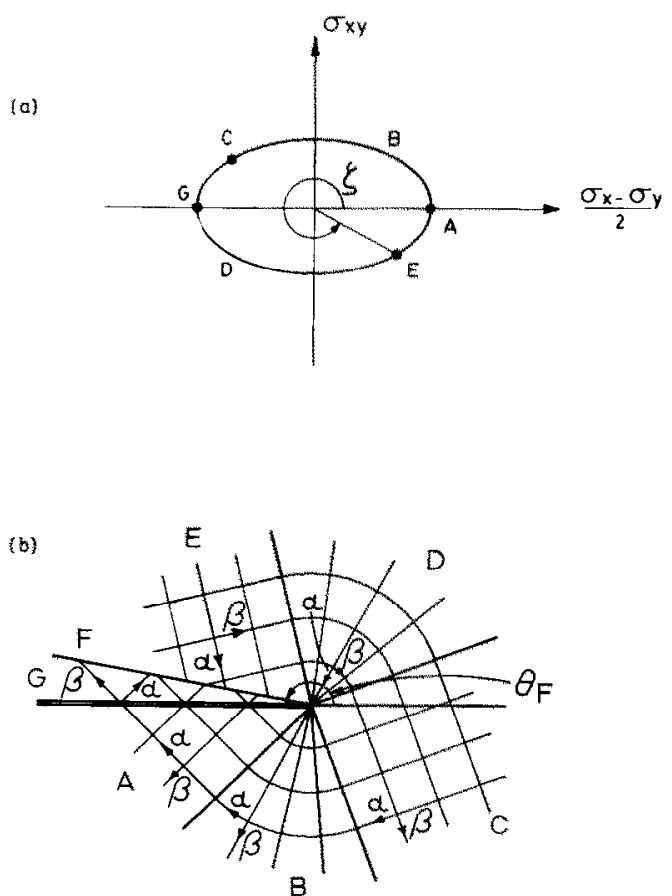

Fio. 7. (a) The elliptical yield contour. (b) The mixed mode crack-tip slip-line field with stress discontinaity. 
At this moment we will use the Mode I stress field as shown in Fig. 3 as the basis to construct the mixed Mode I and II stress field. As shown in Fig. 3a for the Mode I case, point $E$ shares the same position with point $A$. We consider the loading conditions where a small load, which alone would produce a Mode II crack-tip field, is combined with a dominant load which alone would produce a Mode I crack-tip field. To generate a possible mixed mode solution we move point $E$ (corresponding to the constant stress sector $E$ at the crack tip) on the yield contour as shown in Fig. 3 a clockwise to a position as shown in Fig. 7a. The amount of the move depends upon the ratio of the Mode II load to the Mode I load. Point $C$ (corresponding to the constant stress sector $\mathrm{C}$ at the crack tip) consequently has to move clockwise from the position as shown in Fig. 3a to the position as shown in Fig. 7a in order to satisfy the equilibrium conditions. Since the stress state on the upper crack face has to correspond to the stress state of point $G$, we therefore expect that a jump of the radial stress may occur.

Figure 7 shows the complete picture of the mixed mode stress field. Below the lower crack face we have the constant stress sector A corresponding to the stress state of point $A$ on the yield contour. Sector A has the span of angle of $\pi / 4$. Then we have the fan sector $B$ corresponding to the stress state along the yield contour from point $A$ to point $C$ as shown in Fig. 7a. The angle of span of sector B depends upon the exact position of point $C$ on the yield contour.

Adjacent to sector $\mathrm{B}$, we have the constant stress sector $\mathrm{C}$ (corresponding to the stress state of point $C$ on the yield contour) with the angle of span of $\pi / 2$. Then we have the fan sector $D$ which represents the stress state along the yield contour from point $C$ to point $E$. The angle of span depends upon the positions of point $C$ and point $E$ on the yield contour.

Then we have the constant stress sector E corresponding to the stress state of point $E$ on the yield contour. Finally we have the constant stress sector $\mathrm{G}$ corresponding to the stress state of point $\dot{G}$ on the yield contour. Since point $E$ and point $G$ have different positions on the yield contour, the stress states are different. However, the continuity of the traction along the border line $F$ between the two sectors gives

$$
\begin{aligned}
& \sigma_{\theta \theta}^{\mathrm{E}}=\sigma_{\theta \theta}^{\mathrm{G}}, \\
& \sigma_{r \theta}^{\mathrm{E}}=\sigma_{r \theta}^{\mathrm{G}} \quad \text { on the border line } F,
\end{aligned}
$$

where the stress with a superscript "E" represents the stress on the side of sector E and one with a superscript " $G$ " represents the stress on the side of sector G. Consequently the radial stresses $\sigma_{r r}$ are discontinuous across the border line $F$. Note that the directions of the $\alpha$ and $\beta$ lines in each sector are shown in Fig. 7b. From equations (3.2) and (2.1), we can solve for the angle of the border line $F, \theta_{F}$, and the exact position of point $C$ for a given position of point $E$.

For example we select point $E$ such that the angle $\zeta$ of the radial line which passes through point $E$ is $315^{\circ}$ as shown in Fig. 7a. The results of the crack-tip stress field are plotted in Figs. $8 \mathrm{a}-\mathrm{e}$ for $p=10,2,1,0.5$ and 0.1 , respectively. Note that the stresses shown in Figs. $8 \mathrm{a}-\mathrm{c}$ are normalized by $\sigma_{e}$ and the stresses labelled with the "bar" shown in Figs. 8d and e are normalized by $\sigma_{e} / p^{1 / 2}$. When the elliptical yield contour becomes circular in the Mohr plane for isotropic plastic materials, the quali- 

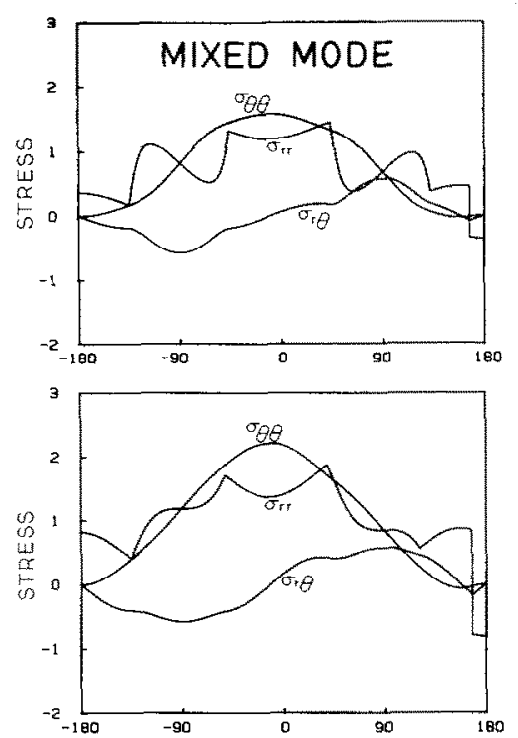

(a)

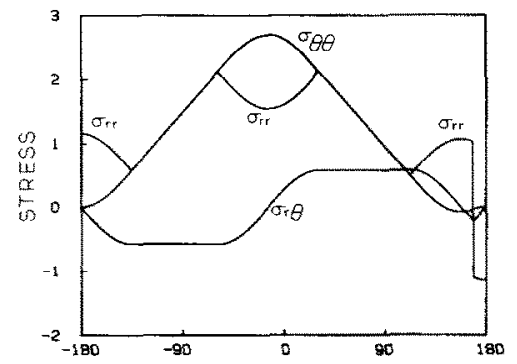

(b)

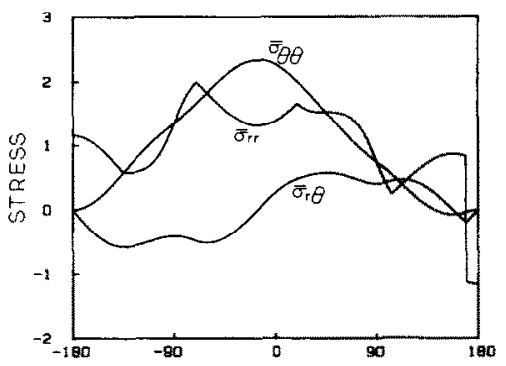

(c)

(d)

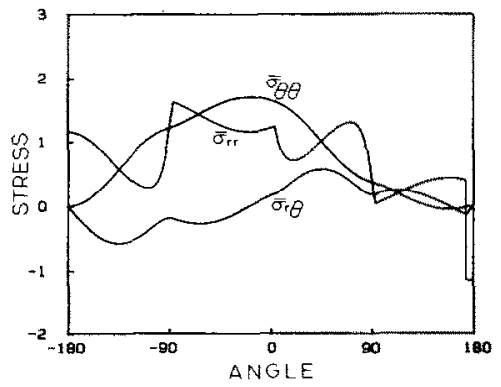

(e)

Fig. 8. The solutions of the mixed Mode I and II crack-tip stress feld with stress discontinuity for $\zeta=315^{\circ}$; (a) $p=10$, (b) $p=2$, (c) $p=1$, (d) $p=0.5$, (e) $p=0.1$. 
tative features of the crack-tip stress field as shown in Fig. $8 \mathrm{c}$ agree with those presented in SHIH $(1973,1974)$. Because of the different stress states in the constant stress sectors $\mathrm{E}$ and $\mathrm{G}$, we can see the discontinuity of the radial stress in these figures.

For a crack in a ductile material under mixed mode loading conditions, the mean in-plane stress and the hoop stress near the tip are important parameters for crack initiation and growth. Note that under plane-strain conditions the out-of-plane normal stress depends upon the details of the three-dimensional yield condition for the orthotropic plastic materials, see HILL (1950) and PAN and SHIH (1986). By comparing Figs. 4a-e with Figs. 8a-e, we found that for mixed mode crack-tip field the maximum mean in-plane stress and the maximum hoop stress are less than those associated with the corresponding pure Mode I crack-tip field. This can be explained easily when we compare the arc length from point $A$ to point $C$ in Figs. 3a and 7a. The constant stress sector $C$ corresponding to point $C$ has the maximum mean in-plane stress around the crack tip. The increase of the mean in-plane stress from point $A$ to point $C$ equals the arc length from point $A$ to point $C$. This qualitative result can be obtained from equation (2.1) for the $\alpha$ lines which are shown in Figs. 3b and $7 \mathrm{~b}$. For the mixed mode crack-tip field, point $C$ is moved clockwise along the yield contour as point $E$ is moved from the position of point $A$ when the crack-tip field deviates from the Mode I stress ficld. As the Mode II contribution under mixed mode conditions increases, the arc length $A C$ decreases; the maximum mean in-plane stress and the maximum hoop stress therefore decrease.

When point $E$ moves to the position of point $G$ as the Mode II contribution becomes large, the radial stress discontinuity on the border line $F$ disappears. This mixed mode crack-tip field has the slip-line field similar to the Mode II crack-tip field shown in Fig. $5 b$ but with sector $F$ degenerated to a line and different span angles for sectors B and D when compared to those of the Mode II crack-tip field. When we further move point $E$ clockwise as the Mode II contribution increases further, the degenerated sector $\mathrm{F}$ expands. We will have the mixed mode crack-tip field similar to the Mode II crack-tip field but the span angles of sectors B, D and F are different from those of the Mode II crack-tip field. When we further move point $E$ clockwise to the position where point $E$ and point $C$ are symmetric with respect to the $\sigma_{x v}$ axis, the crack-tip stress state will reach the anti-symmetric Mode II crack-tip field.

In summary, by moving point $E$ from the position of $A$ shown in Fig. 3 a to the position as shown in Fig. $5 \mathrm{a}$, we can generate a complete range of mixed mode cracktip fields. The solutions for the stress field can be obtained easily by the Rice planestrain slip-line theory. However, the crack-tip stress solutions for the perfectly-plastic orthotropic materials under mixed mode conditions obtained here should be correlated to the perfectly-plastic limit of the crack-tip stress solutions for the correresponding power-law hardening materials.

\section{Discussion}

As in Rice (1973), we note that the mean in-plane stress in front of a Mode I crack tip equals the semimajor (or semiminor) axis $a\left(=\tau_{e} / p^{1 / 2}\right)$ plus half of the 
circumference of the ellipse. As $p$ decreases, $a$ and the circumference of the ellipse increase. Consequently, the mean in-plane stress ahead of the Mode I crack tip increases. This is shown in Figs. 4a-e. Note that the stresses labelled with the "bar" shown in Figs. $4 \mathrm{~d}$ and $\mathrm{e}$ are normalized by $\sigma_{\mathrm{e}} / p^{1 / 2}$.

For a Mode I crack, the fan sectors in which the displacement gradient has a $1 / r$ singularity have the angle of span of $\pi / 2$. The reason is that, as shown in Fig. 3a, the directions of the normals at point $A$ and point $C$ on the yield contour differ by $\pi$. Therefore the angle of span for the fan sectors of the Mode I crack-tip field in the orthotropic plastic materials is the same as that associated with the Mode I crack-tip field in isotropic plastic materials.

As in PAN and SHIH (1986) implications of the present crack-tip field to the fracture analysis of fiber reinforced materials are discussed below. Note that the discussions for composite materials are valid when the characteristic sizes of the fibers such as the fiber diameter as well as the fiber spacing are small compared to the relevant macroscopic dimensions of the composite structure such as the plastic zone size, physical dimensions of the component and other characteristic macroscopic lengths.

We consider a crack in an anisotropic material as shown in Fig. 2 where a family of fibers is reinforced in the $\omega$ direction (as shown in the insert) in an otherwise isotropic material. We consider the case where the crack line is perpendicular to the fiber reinforcement direction $(\omega=0)$. In this case the tensile strength of the reinforced material in the $y$ direction may increase significantly; however,the tensile strength in the $x$ and $z$ direction and the shear strength with respect to the $x, y$ axes may not change significantly. When plastic flow is considered as the deformation mechanism, we can experimentally measure two plane-strain parameters, $a$ and $b$, where $a$ represents the shear yield strength of the material in the direction $\theta=\pi / 4$ and $b$ represents the shear yield strength of the material in the direction $\theta=0$. As discussed in RICE (1973), when the yield contour of the composite material is convex, a yield contour of rectangular shape as shown in Fig. 10a may be an upper bound for the yield contour of the material and the yield contour of diamond shape as shown in Fig. 11a may be a lower bound for the yield contour of the material. For a simple estimation we can choose an elliptical yield contour with the semimajor axis $a$ and semiminor axis $b$ as the yield criterion for the composite material. The yield contours of the materials of this class can be described by equation (3.1) with $p<1$.

Therefore the solutions in Figs. $4 \mathrm{~d}$ and $\mathrm{e}$ for $p=0.5$ and 0.1 represent the Mode I crack-tip stresses for the materials of this class. As discussed earlier, the crack-tip stresses ahead of the crack tip increase as $p$ decreases. For $p=1,0.5$ and 0.1 , ahead of the crack tip the values of the mean in-plane stress $\sigma / \sigma_{e}$ are 2.39, 3.02 and 5.85, respectively; the values of the hoop stress $\sigma_{\theta \theta} / \sigma_{e}$ are 2.97,3.84 and 7.67, respectively; the values of the radial stress $\sigma_{r r} / \sigma_{e}$ are $1.81,2.21$ and 4.03 , respectively.

Note that $\sigma_{e}=3^{1 / 2} \tau_{e}$ where $\tau_{e}(=b)$ is the material shear yield strength with respect to the $x, y$ axes. When we reinforce the materials by strong fibers in the $y$ direction we significantly increase the value of $a$ (the semimajor axis) and may increase the value of $b$ (the semiminor axis); as discussed above, we also significantly increase the crack-tip stresses (normalized by the increased $b$ ) of a Mode I crack which may exist in these materials. The large macroscopic crack-tip stresses may cause a great deal of local damage such as fiber breakage, fiber pullout, dccohesion between the 
matrix and the fiber, and failure of the matrix in these high strength composite materials.

Next we consider a composite material with a family of fibers in the $\omega$ direction as shown in the insert of Fig. 2. We assume the yield contour of the composite material in the Mohr plane is elliptical. The yield contour in the Mohr plane for this case is shown in Fig. 9a. Compared to the contour shown in Fig. 3a the yield contour shown in Fig. 9a has a counterclockwise rotation by $2 \omega$ in the Mohr plane. Similar to the symmetric Mode I case with $\omega=0$, we can construct a slip-line field at the crack tip. The result is shown in Fig. $9 \mathrm{~b}$. This is a possible crack-tip field where the shear stress ahead of the crack tip disappears, but the stress field is not symmetric with respect to the crack line as the conventional symmetric Mode I stress field.

Similarly, we can construct a slip-line field around the crack tip corresponding to point $A$ to point $C$ on the yield contour as for the anti-symmetric Mode II case with $\omega=0$. But the stress field constructed in this manner is not exactly anti-symmetric as is the conventional anti-symmetric Mode II stress field.

For a given ratio of $\sigma_{r \theta}$ to $\sigma_{\theta \theta}$ ahead of the crack tip, we can find a corresponding stress field using the method for constructing the conventional mixed Mode I and II crack-tip stress field as presented earlier. As noted before the stress field may involve radial stress discontinuity.

Now let us discuss some interesting properties due to the geometric symmetry of the elliptical yield contours. When the direction of fiber reinforcement is rotated by $\omega$, the yield contour in the Mohr plane is rotated by $2 \omega$. Consider material A with $\omega=0$ and material B with $\omega=n \pi / 4(n=1,3)$. The yield contour of material A has

(a)
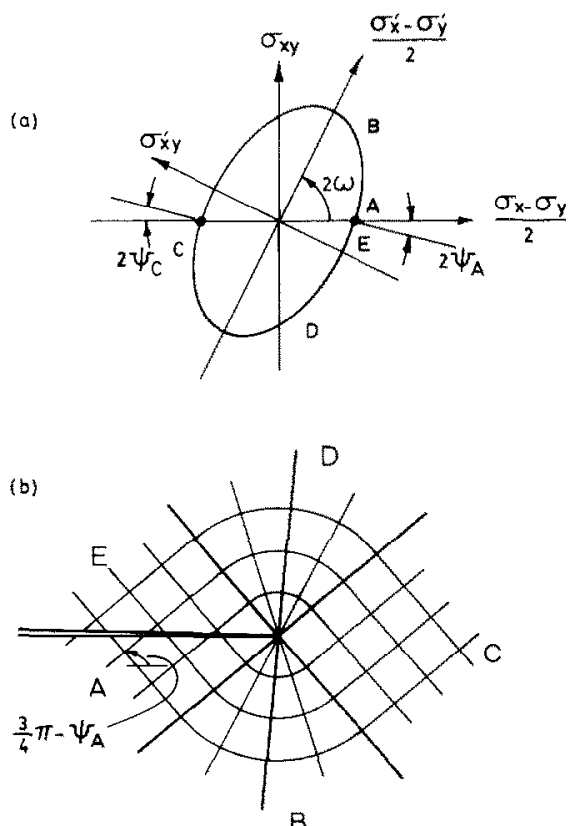

$B$

Fic. 9. (a) The elliptical yield contour. (b) The "Mode I" crack-tip field. 
the general shape described by equation (3.1) with $p<1$. Material $\mathrm{B}$ has the yield contour described by equation (3.1) with $p>1$. The crack-tip stress distribution of material $\mathrm{B}$ then can be estimated using the solutions for $p>1$. The general features of the slip-line field near the crack tip under Mode I, Mode II and mixed Mode I and II conditions for materials A and B are the same. However, the angular variations of the stresses for material $\mathbf{B}(p>1)$ are quite different from those for material $\mathbf{A}(p<1)$, as shown in Figs. 4a-e, 6a-e and 8a-e.

When we reinforce the material by fibers in the direction of $\omega=\pi / 2$ (in the $x$ direction), because of the symmetry of the ellipse we have the same yield condition as that of the material with $\omega=0$. The crack-tip stress fields are therefore the same as those for $\omega=0$. In general the rotation of $\pi$ of the yield contour in the Mohr plane corresponds to the rotation of $\pi / 2$ for the material in the physical plane. Therefore, under a given loading condition the same stress field at the tip of a crack prevails when we reinforce the material either in say, the $\omega_{1}$ direction or in the $\omega_{1}+\pi / 2$ direction when we assume that the yield contour is elliptical in the Mohr plane. This qualitative feature for incompressible materials with the reduced yield condition was noted in the studies of HILl (1950) and PAN and SHIH (1986).

As mentioned earlier, a yield contour of rectangular shape can be used as an upper bound for the yield contour of the composite materials. A yield contour of diamond shape can be used as a lower bound for the yield contour of the composite materials. We can construct crack-tip fields for the yield contours mentioned above according to the method in RiCE (1973). As shown in Fig. 10b, the Mode I crack-tip field for the rectangular yield contour has seven constant stress sectors with radial stress

(a)

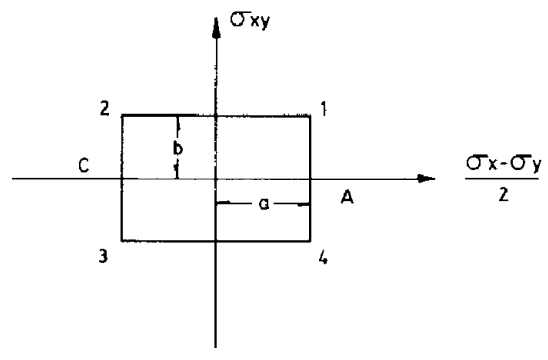

(b)

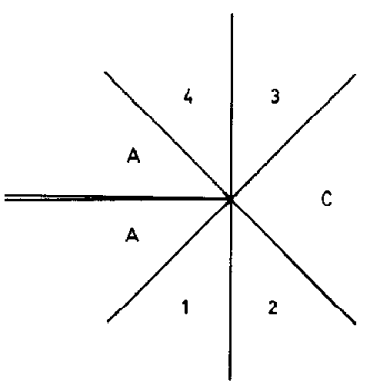

FIG. 10. (a) The rectangular yield contour. (b) The Mode I crack-tip field. 
discontinuity along the borders between the sectors. The sectors labelled with $\mathrm{A}, 1$, 2, C, 3, 4, A have constant stress states corresponding to the stress states of point $A$, $1,2, C, 3,4, A$ on the yield contour as shown in Fig. 10a. As shown in Fig. $11 \mathrm{~b}$ the Mode I crack-tip field for the diamond yield contour has five constant stress sectors with radial stress discontinuity along the borders between the sectors. The sectors labelled with $\mathrm{A}, \mathrm{B}, \mathrm{C}, \mathrm{D}, \mathrm{A}$ have constant stress states corresponding to the stress states of points $A, B, C, D, A$ on the yield contour as shown in Fig. 11a. The details of the construction of the crack-tip field for single crystals with this kind of polygonal yield contour can be found in RICE $(1973,1986)$.

Similar to the remarks in RICE (1973) for punch problems, the mean in-plane stress ahead of a Mode I crack tip is the shear strength for the orientation $\theta=\pi / 4$ plus half of the circumference of the yield contour. Therefore, the estimation of the mean inplane stress ahead of a crack tip under Mode I conditions lies between $a+2\left(a^{2}+b^{2}\right)^{1 / 2}$ for the diamond shape yield contour and $3 a+2 b$ for the rectangular shape yield contour. Note that $a$ represents the shear yield strength in the direction $\theta=\pi / 4$ and that $b$ represents the shear yield strength in the direction $\theta=0$. The simple estimation formula may be useful for the design of fracture-resistant composite materials.

RICE (1986) studied the crack-tip field for stationary and growing cracks when the crack planes are coincident with the symmetry planes in b.c.c. and f.c.c. single crystals. The yield contour for the b.c.c. crystal in Rice's study can be represented by a diamond yield contour as shown in Fig. 11a. However, when the crack plane is not coincident with one of the symmetry planes, the symmetry of the yield contour with respect to the axes of the Mohr plane is not preserved. Then we will not have the conventional symmetric Mode I and anti-symmetric Mode II crack-tip field.
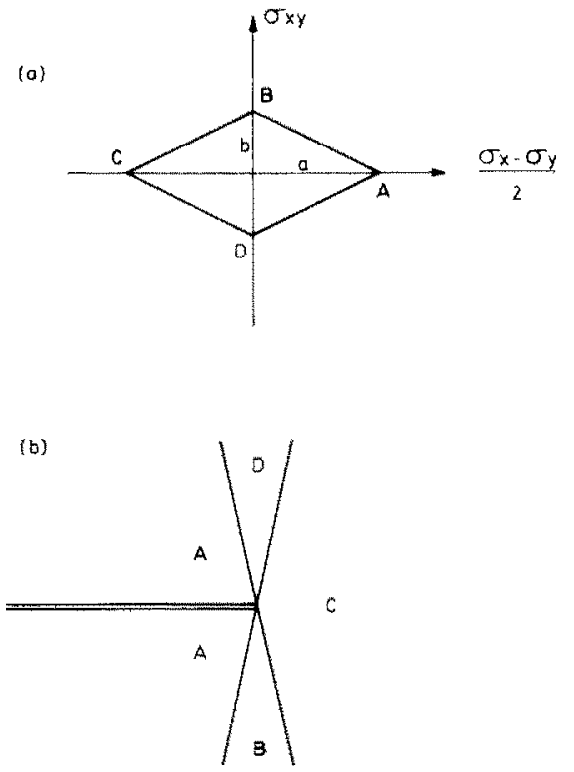

FIG. 11. (a) The Diamond yield contour. (b) The Mode I crack-tip field. 


\section{ACKNOWLEDGEMENT}

Helpful discussions with Professor C. F. Shih of Brown University and Professor J. R. Rice of Harvard University are greatly appreciated.

\section{REFERENCES}

Bishop, J. F. W. and HiLl, R. BUDIANSKY, B and RICE, J. R. HAYASHI, K.

HiLL, R.

HILl, R.

Huichinsun, J. W. Hutchinson, J. W.

PAN, J. and SHIH, C. F. RICE, J. R.

RICE, J. R.

RICE, J. R.

Rice, J. R. and Rosengren, G. F. SHIH, C. F.

SHIH, C. F.
1951 Phil. Mag. 7, 414.

1973 J. appl. Mech., 40, 201.

1979 J. Mech. Phys. Solids 27, 163.

1948 Proc. R. Soc. A193, 281.

1950 The Mathematical Theory of Plasticity, Oxford University Press, London.

1968 a J. Mech. Phys. Solids 16, 13.

1968b J. Mech. Phys. Solids 16, 337.

1986 Mechanics of Materials (in press).

1968 J. appl. Mech. 35, 379.

1973 J. Mech. Phys. Solids 21, 63.

1986 Private communication, to be published.

1968 J. Mech. Phy.s. Solids 16, 1.

1973 "Elastic-plastic analysis of combined mode crack problems", Ph.D. thesis, Harvard University.

1974 Fracture Analysis, ASTM STP 560, p. 187, American Society for Testing and Materials, Philadelphia. 